\title{
Channel-Reuse for Accumulative Repetition Message-Forwarding on Wireless Ad-Hoc Networks
}

\author{
Kelvin K. Lai, Roger S. Cheng, Member, IEEE, Albert K. Wong, Member, IEEE \\ Department of Electronic and Computer Engineering \\ The Hong Kong University of Science and Technology \\ Email: \{kmlai, eecheng, eealbert $\}$ ust.hk
}

\begin{abstract}
Maximizing the rate of transmission over a network with fixed amount of power gives the most economic way to communicate in a network. This paper investigates accumulative repetition forwarding strategy in a wireless ad-hoc network using cooperative forwarding approaches that allow adaptive power allocation among nodes. We analyze the feasibility and performance gain of channel reuse over this network, where multiple transmit-and-receive pairs coexist simultaneously using the same channel. Under this framework, we present the jointly optimal power allocation and channel reuse solution. This solution can be computed in polynomial time and brings significant improvement in network performance.
\end{abstract}

\section{INTRODUCTION}

Communications over wireless ad-hoc network has received great interests in the last couple years and the performance measures to be optimized include capacity, power and bandwidth efficiency. Various cooperative message forwarding and combining strategies had been proposed, including Amplifyand-Forward (AF), Decode-and-Repeat (DR) and Decode-andForward (DF). They are different in the code complexity. Relay nodes in AF only forward an amplified version of received signal while DR requires relay nodes to decode the original message and then regenerate it. For DF, relay nodes decode the message and then re-encode the message before sending to next hop. The re-encoded message is not necessarily identical to the received message, nodes can use different codebooks to encode a message.

Using cooperative message forwarding strategy has great potential for performance improvement. The maximum achievable capacities on wireless ad-hoc network using DR and DF were studied in [1]. Using network coding which is a generalized version of Decode-and-Forward, [2] investigated the minimum energy per bit treating both capacity and power consumption as optimization parameters in the wireless ad-hoc network. The minimization of the transmit power under the assumption of orthogonal transmissions was studied in [4], in which the optimal parallel-relay channel power allocation for AF and DF were derived. Similarly, the problems of optimal resource allocation for DF and Compress-and Forward (CF) were studied in [6].

The greater interest of wireless ad-hoc network problem stems from wireless multicast advantage [3], where signal propagation naturally to multiple nearby nodes simultaneously. Since a single transmission is possible to be received by multiple nodes in the signal coverage, signal can be viewed as broadcast signal and multiple receiving nodes receive the transmission at different power levels. In a DR network, if a receiving node that has sufficient receive power level will decode, and repeat forwarding the message, other receiving nodes with lower receive power level in the first transmission can now benefit from the subsequence retransmissions. Although the receive power level in each transmission may not be large enough, the combined power in multiple transmissions will accumulate to a level enough for reliable decoding. This concept was illustrated in [3] and was referred to as accumulative broadcast. Accumulative broadcast makes use of the unreliable overheard information to increase the network capacity and energy efficiency, because the network topology ensures nodes to receive multiple copies of the same transmission as a data packet is forwarded through the network.

In this paper, we propose a channel reuse scheme to achieve extra performance gain on top of accumulative broadcast. While a weak transmission received by a distant node contributes to the final accumulated power in that node, it may be better to treat this weak received signal as interference and reuse the frequency channel and/or time slot to transmit another packet. Under this framework, extra spatial bandwidth can be created by spatial separation, and nodes share the same channel and transmit simultaneously. We jointly optimize the channel reuse schedule and the power allocation in a wireless ad-hoc DR network employing accumulative broadcast under a rate constraint.

The rest of this paper is organized as follows. Section II introduces the system model and preliminaries used. Section III gives a brief description of maximum capacity and minimum total power consumption solution in the network. Section IV describes and analyzes the usual TDMA scheme and the proposed Channel-Reuse (ChR) scheme, thereafter presents a capacity-achieving minimum total power allocation algorithm. Section V shows the performance gain of $C h R$ over the usual TDMA scheme which does not have channel reuse. Finally, we conclude the paper in Section VI.

\section{System Model AND PREliminaries}

We consider a general wireless chain network which consists a source-and-destination pair, and relay nodes are placed between the source and destination for forwarding information. This simple model is shown in Fig.1. The source $S$ sends out information and relay nodes help delivering that message 
to the destination $D$. Network routing strategies, e.g shortest path, can be used to find a route from the source to the destination and the relay nodes along the route naturally form a connected chain network. In particular, we consider

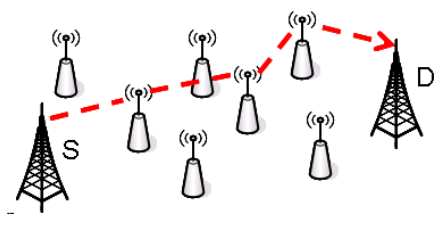

Fig. 1. Wireless Chain-Relay Network

a chain network which consists of $n+1$ nodes with a single source-and-destination pair at both ends. We index the source and destination as 0 and $n$ respectively and the relay nodes according to the order of message forwarding along the route. We defines a symmetric $n \times n$ distance matrix $\mathbf{D}$, in which $d_{j, i}$ is the distance separation between node $j$ and $i$. We assume nodes using sector antenna such that signal only propagates in the direction towards the destination. Also nodes operate in half-duplex mode; when a node is transmitting, it does not receive. We do not consider fading and thus the channel is modeled by a time-invariant AWGN channel with a constant gain according to the path loss model. In each time slot, node $j$ observes a noisy version of input $X_{i}$ from node $i$

$$
Y_{j}(i, D)=\sqrt{E_{i} h_{j, i}} X_{i}+Z
$$

where $E_{i}$ is the transmitted signal energy of node $i ; Z$ is the AWGN with power spectral density level $N_{0}$; and $h_{j, i}$ characterizes the path loss from node $i$ to node $j$, it follows the path loss model and can be written as

$$
h_{j, i}=\left(\frac{d_{j, i}}{d_{0}}\right)^{-\delta}
$$

where $\delta$ is the path loss exponent and the gain is normalized to the path loss at the reference distance $d_{0}$ (i.e. $h_{j, i}=1$ when the distance is $d_{0}$ ). Hence, the receiver Signal-to-Noise Ratio (SNR) at node $j$ of a signal from node $i$ is given by

$$
\gamma_{j, i}=\frac{P_{i}}{N_{0}} h_{j, i}
$$

where $P_{i}$ is the transmit signal power at node $i$.

We assume the system uses DR strategy under the framework of accumulative broadcast, we further refer this as Accumulative Repetition Forwarding (ARF). ARF simplifies both system architecture and coding complexity, it also allows us to benefit from the unreliable overheard information. We start by considering a chain network model example with 4 nodes as shown in Fig.2, where node 0 is the information source, node 1 and 2 are relay nodes, node 3 is the destination. The source is the first to transmit a packet. Node 1 decodes and re-transmits the source messages to the next hop; thus node 2 has chance to overhear node 0's signal in the first transmission in addition to the decode-and-repeat from node 1 , it can benefit from jointly decoding the messages by maximum ratio combining (MRC) and replicate that message to node 3 again. When the message finally propagates to the destination, the message will be decoded by MRC. In the whole process, node 3 has three chances to hear the same message.

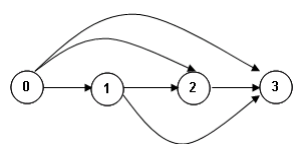

Fig. 2. Wireless Chain-Relay Graph

\section{CAPACITy AND Total Power Consumption}

Network routing strategy imposes a constraint in the message forwarding order in the network. Node is said to be reliable for a message if it has received enough power to decode the message, and it only re-transmits a received message after it became reliable. Node decodes a message by combining all overhead transmissions of a specific set of transmitting nodes which became reliable prior to it. An optimal solution to ARF problem is characterized by a reliability schedule and a power allocation scheme, Reliability schedule specifies the order in which the nodes become reliable. Now given a network route as the reliability schedule, we determine the minimum total power consumption for a required data rate $r$ from the source to the destination.

In each time slot, nodes take turn to transmit in TDMA fashion according to the reliability schedule, we define a power allocation vector $\vec{P}=\left[P_{0}, P_{1}, \ldots, P_{n-1}\right]$ that specifies the transmit power of the nodes. When a node $k$ transmits, it adjusts the transmit power $P_{k}$ to re-transmit the message, so that its succeeding node in the schedule is able to jointly decode the message by MRC. The later nodes in the reliability schedule can receive more copies of the same message, so the equivalent receiver SNR at node $k$, treating the multiple receptions as repetition code, is the sum of the SNRs of all previous overheard transmissions. We generalized the maximal receivable information rate from the source to node $k$ in [1],

$$
R_{k}(\vec{P}, \mathbf{D}, n)=\frac{1}{n} \log _{2}\left(1+\sum_{i=0}^{k-1} \gamma_{k, i}\right)
$$

which is a function of $\vec{P}, \mathbf{D}$ and $n$. The maximal achievable rate of a chain network under ARF regime follows maximum flow minimum cut theorem, is given by [1]

$$
C_{r e p}(\mathbf{D}, n)=\max _{\vec{P}}\left(\min _{k \in[1: n]} R_{k}(\vec{P}, \mathbf{D}, n)\right)
$$

Under the framework of ARF, $\vec{P}$ is defined to be ARF valid for a required data rate $r$ iff

$$
R_{k}(\vec{P}, \mathbf{D}, n) \geq r \forall k \in \boldsymbol{K} \text { and } \vec{P} \succeq 0
$$

where

$$
\boldsymbol{K}=\left\{i \mid i \in[1: n-1], P_{i}>0\right\}
$$

and $\succeq$ is defined as $P_{k} \geq 0 \forall k$.

For an ARF valid power vector, node has to transmit at positive power, the transmission from any node $k$ which has 
$R_{k}(\vec{P}, \mathbf{D}, n)<r$ is useless, because itself cannot decode the message correctly, the subsequence nodes in the reliability schedule thus cannot include node $k$ 's transmission to decode the message, the energy collected from node $k$ should be excluded from the link capacity calculations using (4). Instead, a power allocation vector which is not ARF valid is $A R F$ valid convertible. It means an ARF invalid power allocation vector is always able to be converted into ARF valid. It is important to note that $R_{k}(\vec{P}, \mathbf{D}, n)$ only depends on $P_{0}, \ldots, P_{k-1}$. If we change the amount of power allocated to node $k$, only the maximal achievable information rate $R_{l}(\vec{P}, \mathbf{D}, n)$, where $l>$ $k$, will be affected. The conversion mechanism is that when we find any node $\mathrm{k}$ which has $P_{k}>0$ and $R_{k}(\vec{P}, \mathbf{D}, n)<r$, we simply set $P_{k}=0$ because any transmission from node $k$ is not reliable at any rate. We better shutdown node $k$ to save power, it still preserves the capacity achieved by the original power allocation vector.

Given any power allocation vector $\vec{P}$, we can convert it to ARF valid $\vec{V}$. The following gives a constructive algorithm.

1) ARF_Conversion_Algorithm $(\vec{P}, \mathbf{D}, n, r)\{$

$\vec{V}=\vec{P}$

2) FOR $k=0$ TO $n-1$

3) $\quad \operatorname{IF}\left(V_{k}>0\right)$ AND $\left(R_{k}(\vec{V}, \mathbf{D}, n)<r\right)$

4) $\quad V_{k}=0$

5) update $R_{l}(\vec{V}, \mathbf{D}, n) \forall l>k$

6) END IF

7) END FOR

8) RETURN $\vec{V}\}$

The ARF Conversion Algorithm always returns an ARF valid power allocation vector. If the input vector $\vec{P}$ is already ARF valid, then $\vec{V}=\vec{P}$. The algorithm does not change the original achieved capacity by $\vec{P}, R_{k}(\vec{V}, \mathbf{D}, n)$ is always equal to $R_{k}(\vec{P}, \mathbf{D}, n)$, the conversion restricts the transmit power is only allocated to the nodes which can reliably decode the message, in order to comply the ARF assumptions.

Besides the maximal achievable capacity, it is also common to find the minimum cost of transmitting data at a given rate $r$. The cost can be measured in different quantities, e.g. total power consumption, bandwidth, spectrum efficiency etc. In wireless ad-hoc network, power is always the main concern because the system is typically assumed to be batterypowered and power limited. The problem of minimum total power consumption is actually a dual problem of maximizing network capacity. Given a required data rate $r$, the minimum total power $P_{\text {Total }}$ required to achieve this is equivalent to the objective that maximizes the data rate $r^{*}$ under a total power constraint $P_{\text {Total }}^{*}$; Such that $P_{\text {Total }}=P_{\text {Total }}^{*}$ if and only if $r=r^{*}$. Theorem 1.2 states the necessary and sufficient conditions for the optimal solution of this dual problem.

Lemma 1.1: Given a required information rate $r$ and a power allocation vector $\vec{P}$, the destination can successfully receive the information from the source using ARF if and only if $R_{n}(\vec{V}, \mathbf{D}, n) \geq r$ where $\vec{V}=$ ARF_Conversion_Algorithm $(\vec{P}$, $\mathbf{D}, n, r)$.

Proof: A node always tries to decode a message by combining the overheard transmissions from the nodes which had transmitted before, and then regenerates the received message. $\vec{V}$ is an ARF valid vector returned from ARF Conversion Algorithm, it ensures all intermediate transmit nodes would receive enough power to decode the source information. If $\vec{V}$ results $R_{n}(\vec{V}, \mathbf{D}, n) \geq r$, the required date rate is not larger than the link capacity from the source to the destination by $\vec{P}$. Every node $k \in \boldsymbol{K}$ including the destination node can receive and decode the message. In the contrary, if $R_{n}(\vec{V}, \mathbf{D}, n)<r$, the link capacity from the source to node $n$ is less than $r$, it implies the destination is not able to decode the message correctly. Hence, a power allocation vector for overall successful transmission using ARF has to guarantee $R_{n}(\vec{V}, \mathbf{D}, n) \geq r$.

Theorem 1.2: A power allocation vector $\vec{P}$ is optimal with minimum total power consumption $Q_{\text {Total }}$ for a required information rate $r$, if and only if $\vec{P}$ is ARF valid and

$$
R_{k}(\vec{P}, \mathbf{D}, n)=r \forall k \in \boldsymbol{K} \cup\{n\}
$$

Proof: The ARF conversion algorithm either set $V_{k}=P_{k}$ or $V_{k}=0$. If $\vec{P}$ is not ARF valid, there exists at least a node $j$ which has $R_{j}(\vec{P}, \mathbf{D}, n)<r$ and $P_{j}>0$, we can always find an ARF valid vector $\vec{V}=A R F \_C o n v e r s i o n \_A l g o r i t h m(\vec{P})$, such that the sum of $V_{i}$ s is less than the sum of $P_{i}$ s because $V_{j}$ is set to 0 . Although $\vec{P}$ and $\vec{V}$ will result the same network capacity, the total power consumption of $\vec{V}$ is less than those of $\vec{P}$, thus the power allocation vector for minimum total power consumption has to be ARF valid. By Lemma 1.1 and the ARF valid requirement for successful transmission, we have

$$
\begin{array}{rlr} 
& R_{k}(\vec{P}, \mathbf{D}, n) \geq r \quad \forall k \in \boldsymbol{K} \cup\{n\} \\
\Leftrightarrow & \frac{1}{n} \log _{2}\left(1+\sum_{i=0}^{k-1} \gamma_{k, i}\right) \geq r \quad \forall k \in \boldsymbol{K} \cup\{n\} \\
\Leftrightarrow & \mathbf{G}^{\prime} \vec{P}^{\prime} \succeq\left(2^{n r}-1\right) \overrightarrow{1} &
\end{array}
$$

where $\mathbf{G}^{\prime}$ and $\vec{P}^{\prime}$ are punched matrices of $\mathbf{G}$ and $\vec{P}$ respectively. $\mathbf{G}$ is $n \times n$ matrix:

$$
\mathbf{G}=\frac{1}{N_{0}}\left[\begin{array}{cccc}
h_{1,0} & 0 & \ldots & 0 \\
h_{2,0} & h_{2,1} & & \vdots \\
\vdots & & \ddots & 0 \\
h_{n, 0} & \ldots & h_{n, n-2} & h_{n, n-1}
\end{array}\right]
$$

$\mathbf{G}^{\prime}$ is formed by deleting the $j^{\text {th }}$ row and column in $\mathbf{G}$, while $\vec{P}^{\prime}$ is created by deleting $P_{j}$ from $\vec{P}$, where $j \in \boldsymbol{J}=\{i \mid i \in[1$ : $n], i \notin \boldsymbol{K} \cup\{n\}\}$. $\mathbf{G}^{\prime}$ is always invertible, so the inequalities in (9) is equivalent to

$$
\vec{P}^{\prime} \succeq\left(2^{n r}-1\right) \mathbf{G}^{\prime-1} \overrightarrow{1}
$$

and the total power consumption is

$$
\begin{aligned}
P_{\text {Total }}=\overrightarrow{1}^{T} \vec{P} & =\overrightarrow{1}^{T} \vec{P}^{\prime} \\
& \geq\left(2^{n r}-1\right) 1^{T} \mathbf{G}^{-1} \overrightarrow{1}=Q_{\text {Total }}
\end{aligned}
$$

because $P_{j}=0 \forall j \in \boldsymbol{J}$. All $\vec{P}$ satisfying the information rate $r$ would have total power consumption larger than or equal to 
$Q_{\text {Total }}$, unless (9) is satisfied with equality $\forall k \in \boldsymbol{K}$. On the other hand, if we let $\vec{P}$ satisfy (11) with equality, then (9) will hold with equality and $P_{\text {Total }}=Q_{\text {Total }}$. Hence, the optimal power allocation always ensures that (9) would satisfy with equality $\forall k \in \boldsymbol{K} \cup\{n\}$.

\section{Channel-Reuse Performance Analysis}

We have discussed the maximum rate and minimum total power problem of a general chain network using ARF, solving either one can get the other. The rate achievable code construction requires finding the optimal power allocation vector $\vec{P}^{*}$. In the rest of this paper, we approach these problems from the minimum total power $P_{\text {Total }}$ for a required rate $r$. We propose a frequency reuse scheme and analyze how it works with the network using ARF.

Channel-Reuse, ChR proposes multiple simultaneous transmissions used with ARF; it creates extra spatial bandwidth to the network. The system gains parallel transmissions, even the signal undergoes co-channel interference as well. To provide a quick approach to this quantitative evaluation, we adopt the setting in [1], the chain network stands on a straight line, and the nodes are evenly distributed on the line, the destination is with overall distance of $d$ meters from the source. These assumptions only simplify the mathematics allowing us to compare the network capacity and power consumption of $\mathrm{ChR}$ scheme. It is not inherent to our discussion to any distance matrix $\mathbf{D}$ and nodes topology. The good of using this model is easy to understand the problem, the distance matrix and even the signal attenuation between nodes can be generalized into only three parameters $\delta, d$ and $n$. The distance between node $j$ and node $i$ is

$$
d_{j, i}=(j-i) \frac{d}{n}
$$

Motivated by cellular network, a ChR scheme should bring improvement on spectrum efficiency or maybe the network capacity. ChR scheme suggests that if the reception from distant node is weak, channel reuse is recommended in which the transmit-and-receive pairs separated farther away are allowed to transmit simultaneously using the same channel. The gain of channel reuse may be higher than only overhearing the weak broadcast from faraway transmitters, although the simultaneous transmissions interferes each other and lowers the data rate. However it allows multiple packets being forwarded in the network at a time. While we enjoy the power saving from accumulative broadcast, we should consider channel reuse as well. We introduce a new optimization parameter to the problem, called channel reuse factor $m$, it is a logical boundary of signal spread. We further quantify $m$ in term of number of hops for easier analysis, it means that the farthest node the signal can reach and be regarded as useful is $m$ hops array. The idea is giving a reference measure telling when we can reuse the frequency channel. Such that a reliable node whose target receiver nodes are not in signal coverage of all the other transmitters can start a new transmission.

We describe the model with an example that the network contains 11 nodes as shown in Fig.3. Node 0 is supposed to have a stream of equal-size data packets for node 10 initially. In the packet forwarding process, each relay node $i$ holds some new information for its fellow nodes $j$ where $j>i$. The messages received and stored at a node can be divided into two categories, reliable set and unreliable set. The reliable set contains decoded message, while the unreliable set stores the unreliably overheard messages. A message in the unreliable set requires more receptions to become reliable. In the steady state, a node always has one decoded message in its reliable set, but that message is in the unreliable sets of its fellow nodes. When node $i$ transmits, it re-transmits that message to make that message reliable in the next adjacent node, this mechanism ensures the message is being sent toward the destination.

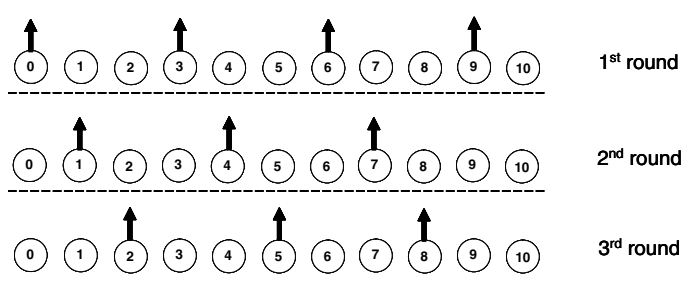

Fig. 3. Transmit schedule for channel reuse factor, $m=3$

In Fig.3, the channel reuse factor $m=3$ divides nodes into three groups, $\{0,3,6,9\},\{1,4,7\}$ and $\{2,5,8\}$. The arrows indicate the nodes are transmitting at a certain time. The data packets are continuously being pumped out from the source (from left to right). Nodes in the same group transmit simultaneously, and groups orderly take turn to transmit in different round. It forms a transmission cycle with $m$ rounds. When node $i$ transmits, nodes $i+1$ to $i+m-1$ are in receiver mode listening to the signal from $i$. In the first round, node 0/3/6/9 transmits a packet, to make node $1 / 4 / 7 / 10$ (next node) reliable, and node $2 / 5 / 8$ overhears a weaker version of the transmission due to it located farther way from the transmitter. In particular, the output to node 4 is an interfered version of the transmissions from node 3 and 0 plus noise, however node 4 only treats node 3's transmission as the informative part, and the transmission from node 0 is regarded as interference. When node 4 is set to be reliable, it will transmit in the next round ( 2 nd round) as what node 3 did, to make node 5 reliable, while node 6 overhears the transmission, and so on in the third round.

A time slot is equally divided into $m$ rounds, node $k$ would have at most $m-1$ rounds in receiver mode in each slot, it listens to the signal from node $i \in\left[u_{k}: k-1\right]$ where $u_{k}=$ $\max [k-(m-1), 0]$. The channel output to node $k$ at a time is a noisy version of input $X_{i}$ with interference:

$Y_{k}(i, d, m)=\sqrt{E_{i} h_{k, i}} X_{i}+\sum_{j=1}^{\left\lfloor\frac{i}{m}\right\rfloor} \sqrt{E_{i-j m} h_{k, i-j m}} X_{i-j m}+Z$

where the first term is the signal from node $i$, second term is the interference due to simultaneous transmissions. So the 
receiver SNIR of node $i$ 's signal is

$$
\gamma_{k, i}=\frac{P_{i} h_{k, i}}{N_{0}+\sum_{j=1}^{\left\lfloor\frac{i}{m}\right\rfloor} P_{i-j m} h_{k, i-j m}}
$$

where the numerator is the signal power and the denominator is the inference plus noise. Hence, the data rate to node $\mathrm{k}$ is

$$
R_{k}^{C h R}(\vec{P}, d, m, n)=\frac{1}{m} \log _{2}\left(1+\sum_{i \in\left[u_{k}: k-1\right]} \gamma_{k, i}\right)
$$

The ChR optimal solution to the minimum total power consumption includes bandwidth and power control. The transmit power of a node cannot be too high, as it may cause too much interference to other simultaneous transmissions. If it is too low, it limits the link capacities itself to the other nodes. Frequency reuse too often also incurs strong signal interference, so we define an object function to maximize the network capacity using $\mathrm{ChR}$ scheme as

$$
C_{r e p}^{C h R}(d, n)=\max _{m}\left[\max _{\vec{P}}\left[\min _{k \in[1: n]}\left(R_{k}^{C h R}(\vec{P}, d, m, n)\right)\right]\right]
$$

given a total power constraint $U_{\text {Total }}$. We approach this problem by solving its dual problem, the minimum total power consumption for a required rate $r$; the corresponding total power consumption is $U_{\text {Total }}$, and hence $C_{r e p}^{C h R}(d, n)=r$.

The following presents a algorithm to solve the problem of minimum total power consumption given $r$, the optimization parameters are $m$ and $\vec{P}$. They can be solved in two phases. we compute the optimal power allocation vector for each $m \in[2$ : $n]$ in the first phase, and the optimal channel reuse factor value can be determined in the second phase. Firstly the optimal power allocation should always satisfy Theorem 1.2 for a given $m$, the proof is omitted here, because it is similar the previous. We can set the capacities to each node equal to $r$ :

$$
R_{k}^{C h R}(\vec{P}, d, m, n)=r \forall k \in[1: n]
$$

(18) is a system of $n$ equations with n unknowns. It is not linear, as the denominator of receiver SNIR contains $P_{i}$ s. We fortunately observed that $R_{k}^{C h R}$ is actually a function $\left\{P_{0}, \ldots\right.$, $\left.P_{k-1}\right\}, P_{i}$ can be computed one by one from $i=0$ to $n-1$, so the whole optimal power allocation vector for a fixed $m$ and $r$ can be obtained in linear time. In the second phase, we select the optimal channel reuse factor $m^{*}$ whose minimum total power consumption is the smallest among all the other $m$. This completes the algorithm by finding the optimal channel reuse factor $m^{*}$ and power allocation $P^{*}$. Indeed the number of nodes $n$ is also a possible optimization parameter in this problem, we can easily modify our objective function and algorithm above with an additional iteration to optimize $n$, such that $C_{r e p}^{C h R}(d)=\max _{n} C_{r e p}^{C h R}(d, n)$, it does not change the time complexity to solve the optimal solution.

\section{Results AnAlysis}

The problem of maximizing the capacity using $\mathrm{ChR}$ in the ad-hoc network is discussed, the optimal channel reuse factor and power allocation are obtainable in polynomial time. This section provides numerical results comparing with the usual TDMA scheme, we considered a network with 40 nodes and $d=100$, the system has total power constraint $P_{\text {Total }}=46 d B W$. We analyzed the network capacity with various number of nodes used and under different path loss exponents. The results of ChR scheme are shown in line with those of TDMA scheme in the follow figures. In addition to the performance gain, it is insightful to understand how the environment factors affect the results and the optimization of ChR scheme.

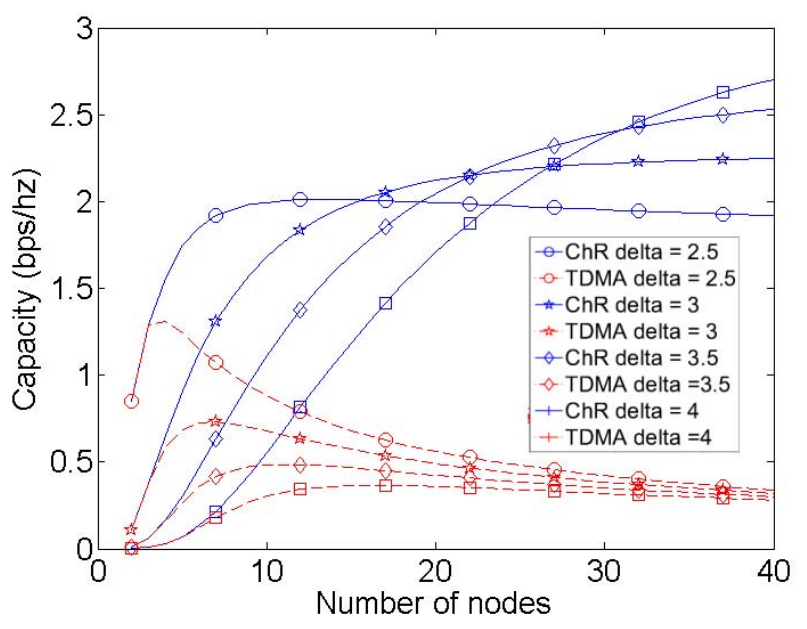

Fig. 4. The maximum achievable rate vs. the number of nodes involved in the transmission at different path loss.

We assumed the case of high SNR $N_{0}=-3 d B W$, Fig. 4 presents the capacity versus the number of nodes used with different path loss exponents. The dashed lines are the plot of capacities of TDMA scheme, while those of ChR scheme are represented in the solid lines, the markers on the lines represent the path loss exponent used in the simulation. Firstly, we focus on the lines of $\delta=2.5$, they originate from the same spot where only 2 nodes are used, it is the simplest case that the source directly transmits to the destination. When more nodes are considered to help forward the messages, the capacities would increase at the beginning, because separation between nodes is shortened, it results the higher receiver SNR so the larger capacities. The TDMA scheme reaches its peak 1.35 $\mathrm{bps} / \mathrm{hz}$ when number of nodes used is 4 , and then its capacity declines continuously with the number of nodes used. The drop is due too many nodes sharing a fixed time slot, the source is only assigned very short time to transmit. A bottleneck is created between the source to the others. In the contrary, $\mathrm{ChR}$ scheme does not inherit this limitation, it allows nodes to transmit simultaneously, it achieves at a much higher date rate. $\mathrm{ChR}$ scheme achieves the maximum $2.01 \mathrm{bps} / \mathrm{hz}$ at 14 nodes, but ChR scheme incurs interference across different transmitand-receive pairs. Placing nodes too close to each other leads to stronger interference, the capacity of $\mathrm{ChR}$ scheme declines with $n$ increase eventually. These adduce the hypothesis above that sometimes less nodes is better. According to Fig.4, using 14 nodes is the best, thus the power is optimally allocated 
only to nodes $\{0,3,6,9,12,15,18,21,24,27,30,33,36,39\}$ and shutdown the others.

As the channel is time varying, so the optimal solution should be reviewed from time to time. The average performance in different environment is a good measurement of a scheme's efficiency. In different path loss environment, ChR scheme significantly improved the capacity over TDMA scheme, the peak-to-peak ratios of ChR scheme and TDMA scheme are $3 \mathrm{~dB}-8 \mathrm{~dB}$. The $\mathrm{ChR}$ scheme performs especially well at high path loss. Although TDMA scheme results somewhat disappointing, it does not exhibit the advantage of having more nodes to relay information, its capacity also generally decreases when path loss increases, it is explainable by the channel capacity theory. In the contrary, ChR scheme performs on average equally well, at about 2-3 bps/hz. Frequency reuse having more parallel channels offsets the effect of high path loss on a particular channel. Two conclusions can be drawn from Fig.4; Firstly, ChR scheme can sustain or exceed the capacity at high path loss environment by using more nodes to relay the information or reusing the channel. On the other hand, the inference dominates and limits the network capacity when the nodes are too close to each other or path loss exponent is small, the peak capacity of small path loss exponent appears at smaller $n$, so that the separation between nodes is sufficiently large to avoid too much interference. High path loss environment is favour with frequency reuse, it cuts down the interference spreading, thereby more simultaneous transmissions can be supported. These explain why the better capacity can be achieved when $\delta$ is large. ChR scheme has an ability that the system can auto-adjust the number of nodes should be used and the frequency reuse in various path loss settings.
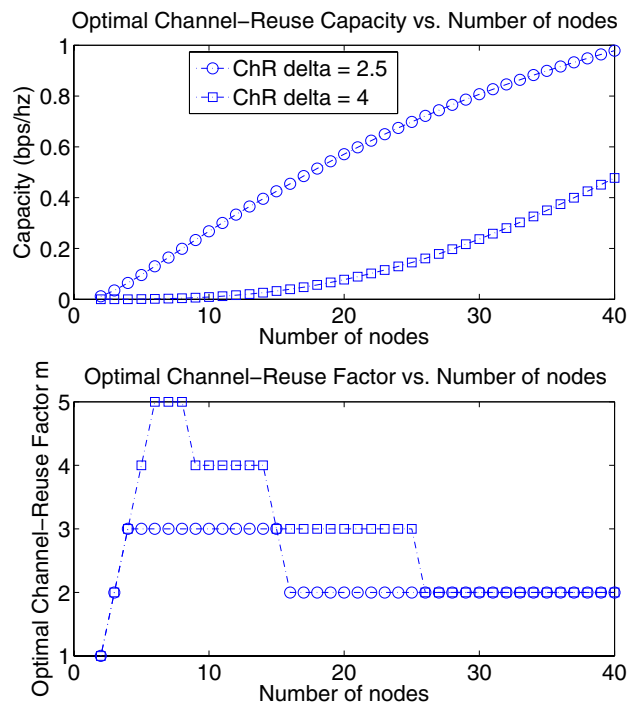

Fig. 5. At low SNR $N_{0}=17 d B W$, the ChR maximum achievable rate vs. the number of nodes and the corresponding optimal channel reuse value $m$. $\delta=2.5$ and 4 .

We find that the $m *$ is always equal to 2 in high SNR cases, the network does not take the advantage of accumulative broadcast, power is used to trade spatial bandwidth. It stimulates our study extended to low SNR case, we continue to use the previous settings except $N_{0}=17 d B W$. Fig.5 shows the results of $\delta=2.5$ and $\delta=4$. The upper figure in Fig.5 shows the maximum achievable rate, and the corresponding $m^{*}$ is plotted in the lower graph accordingly. Lets look at the lines of $\delta=4$, ChR scheme suggests full accumulative broadcast (i.e no channel reuse) when the number of nodes used is less than or equal to 6 , in which the receiver SNR is relatively low because of the large separation between nodes and noise power. ChR scheme decides that the power should be emphasized on each transmission to make it distinguishable from noise and avoids co-channel interference. In view of this, either shorten the separation between nodes or the path loss drops can improve the receiver SNR, consequently $\mathrm{ChR}$ scheme starts to trade spacial bandwidth with power. It is evidenced by Fig.5, the optimal channel reuse value also decrease till $m=2$ when the number of nodes increases or the path loss drops. It further supports the result we obtained previously in Fig. 4 at high SNR. ChR scheme can take a balance between accumulative broadcast and channel reuse.

\section{CONCLUSION}

The paper studies the network capacity problem on an wireless ad-hoc networks using accumulative broadcast in a decode-and-repeat strategy. Accumulative broadcast is optimal in the sense of power saving; however, it does not utilize bandwidth efficiently. We propose the ChR scheme to improve the performance under accumulative broadcast. The proposed scheme combines the merits of both channel reuse and accumulative broadcast to form the accumulative repetition forwarding strategy. With dynamic power allocation and frequency reuse control, the maximum achievable capacity for the proposed scheme is derived. We analyzed the optimal channel reuse and number of relay nodes under various setting, various path loss and SNR levels. We find that the proposed scheme performs much better than the pure accumulative broadcast scheme and believe that this approach can potential increase the wireless ad-hoc network performance significantly.

\section{REFERENCES}

[1] X. Bao and J. Li, "Progressive Network Coding for Message-Forwarding in Ad-Hoc Wireless Network," IEEE SECON, vol. 1, pp. 207-215, Sept 2006.

[2] Y. Wu, P. Chou and S.Y. Kung, "Minimum-Energy Multicast in Mobile Ad Hoc Network Using Network Coding," IEEE Transactions on Communications, vol. 53, pp. 1906-1918, NOV 2005.

[3] J. Wieselthier, G. Nguyen, and A. Ephremides, "On the Construction of Energy-Efficient Broadcast and Multicast Trees in Wireless Networks," IEEE INFOCOM, Nineteenth Annual Joint Conference of the IEEE Computer and Communications Societies, vol. 2, pp. 585-594, Mar 2000

[4] I. Maric and R. Yate, "Forwarding Strategies for Gaussian Parallel-Relay Networks," in Proc. 38th Annu. Conf. Information Sciences and Systems (CISS'04), Princeton, NJ, Mar. 2004.

[5] I. Maric and R. Yate, "Cooperative Multihop Broadcast for Wireless Network," IEEE Journal on Selected Areas in Communications, vol. 22, Issue. 6, pp. 1080-1088, Aug 2004.

[6] G. Kramer, M. Gastpar and P. Gupta, "Cooperative Strategies and Capacity Theorem for Relay Networks," IEEE Transactions on Information Theory, vol. 5, pp. 3037-3063, SEP 2005. 